\title{
Mine Gas Emission Prediction Based on Grey Markov Prediction Model
}

\author{
Yan Wang, Duoxi Yao, Haifeng Lu \\ School of Earth and Environment Anhui University of Science \& Technology, Huainan, China \\ Email:2419668737@qq.com
}

How to cite this paper: Wang, Y., Yao, D.X. and Lu, H.F. (2018) Mine Gas Emission Prediction Based on Grey Markov Prediction Model. Open Journal of Geology, 8, 939-946.

https://doi.org/10.4236/ojg.2018.810056

Received: August 1, 2018

Accepted: September 16, 2018

Published: September 19, 2018

Copyright $\odot 2018$ by authors and Scientific Research Publishing Inc. This work is licensed under the Creative Commons Attribution International License (CC BY 4.0).

http://creativecommons.org/licenses/by/4.0/

\begin{abstract}
This article puts forward the gray markov prediction model to predict mine gas emission by combining grey system theory and markov chain theory. And GM $(1,1)$ model is established in the first place for grey data by the model. To eliminate the error, and improve the prediction accuracy of the model, secondary parameters fitting was done on the basis of GM $(1,1)$ model. And we get second parameter fitting for trend prediction. Then using Markov state transfer probability matrix prediction method to do quadratic fitting for its predictive value, which can improve the prediction precision of the volatile random variables. It proves the prediction results of the model are satisfactory by analyzing history data of gas emission prediction. This conclusion broadens the application scope of grey forecast model and provides a new method for mine gas emission scientific forecast.
\end{abstract}

\section{Keywords}

Parameter Fitting, Grey System, Markov Chain, Gas Emission

\section{Preface}

Gas disaster is one of the most serious disasters in coal mine, the prevention and control of gas has been the focus of every country coal mine safety. Precisely predicting the amount of mine gas emission has important practical significance for guiding the design of mine and production safety. Mine gas emission prediction methods can be divided into mine statistical method, the point source prediction method, the gas gradient method, the mathematical model of coal bed gas content and gas geology, and so on at present [1]-[7]. The above each method has its own application conditions, their prediction processes are static, without considering gas emission is a complicated nonlinear dynamic system [8] [9] [10] [11], so they have difficulties in mine gas emission prediction. Based on 
this, in view of the mine gas emission prediction has a trend and the characteristics of randomness at the same time, this article analyzes the mine gas emission by using grey model and markov prediction model for random process, thus puts forward the gray markov prediction model.

\section{Solution of the Second Parameter Fitting Method of GM $(1,1)$ Model}

Grey system was firstly put forward by Deng julong in 1982 [12]. Grey system refers to the system of information incomplete and uncertain, which is between white and black system. It is used to solve the incomplete information system. Furthermore, it is a combination of automatic control and operational research, and it has penetrated into many fields such as agriculture, economy, transportation, and meteorological and shows a broad application prospect in short more than ten years development. Based on the thought of the known data related to the time combination, which according to certain rules, grey forecasting forms a white model and finally improve the bleaching degree of grey module by some rules. Grey prediction can be divided into season series forecast, disaster forecast, disaster forecast, topological prediction and forecast system whose characteristic is using few data to establish model, but the accuracy is lower the forecasts for stochastic volatility series fitting is poorer.

Single sequence 1 order linear model, one of the series forecast, short for GM $(1,1)$. We set the original observation sequence as $x^{0}(t)(t=1,2, \cdots, n)$. And make a accumulation: $x^{(1)}=\left\{x^{(1)}(1), x^{(1)}(2), \cdots, x^{(1)}(n)\right\}$, in which, $x^{(1)}(k)=\sum_{i=1}^{k} x^{(0)}(i), \quad k=1,2, \cdots, n$

We can establish an albino form:

$$
\frac{\mathrm{d} x^{(1)}}{\mathrm{d} t}+a x^{(1)}=U
$$

In the formula, $a$ and $U$ are undetermined constants.

Then, solving the parameter by means of least square method:

$$
\left(\begin{array}{l}
\hat{a} \\
\hat{u}
\end{array}\right)=\left(B^{\mathrm{T}} B\right)^{-1} B^{\mathrm{T}} Y_{n}
$$

In which,

$$
B=\left[\begin{array}{cc}
-1 / 2\left[x^{(1)}(1)+x^{(1)}(2)\right] & 1 \\
-1 / 2\left[x^{(1)}(2)+x^{(1)}(3)\right] & 1 \\
-1 / 2\left[x^{(1)}(n-1)+x^{(1)}(n)\right] & 1
\end{array}\right], \quad Y_{n}=\left(\begin{array}{c}
x^{0}(2) \\
x^{0}(3) \\
\vdots \\
x^{0}(n)
\end{array}\right)
$$

At the same time, $B^{\mathrm{T}}$ is the transposed matrix of matrix $B$.

Grey prediction equation of $x^{(0)}$ is,

$$
\hat{x}^{(1)}(k+1)=\left(x^{(0)}(1)-\frac{u}{a}\right) \mathrm{e}^{-a k}+\frac{u}{a}
$$

Equation (4) is the time response of GM $(1,1)$ model equation. There are 
large amount of data proved that original data will produce error if we use Equation (4) to fit. In order to improve the fitting precision and prediction precision, we do secondary parameter fitting to Equation (4) [13] and change it into,

$$
\hat{x}^{(1)}(k+1)=\alpha \mathrm{e}^{-a k}+\beta
$$

According to the first estimate of a value $a$ and estimation of original series $x^{(1)}$ to $\alpha$ and $\beta$.

$$
\begin{aligned}
& x^{(1)}(1)=\alpha \mathrm{e}^{0}+\beta \\
& x^{(1)}(2)=\alpha \mathrm{e}^{-a}+\beta \quad \text { written in matrix form is } \\
& \vdots \\
& x^{(1)}(n)=\alpha \mathrm{e}^{-a(n-1)}+\beta
\end{aligned}
$$

$$
x^{(1)}=G\left(\begin{array}{l}
\alpha \\
\beta
\end{array}\right)
$$

In which, $x^{(1)}=\left[x^{(1)}(1), x^{(1)}(2), \cdots, x^{(1)}(n)\right]^{\mathrm{T}}, \quad G=\left(\begin{array}{cc}\mathrm{e}^{0} & 1 \\ \mathrm{e}^{-a} & 1 \\ \vdots & \vdots \\ \mathrm{e}^{-a(n-1)} & 1\end{array}\right)$, according to the least square method,

$$
\left(\begin{array}{c}
\alpha \\
\beta
\end{array}\right)=\left(G^{\mathrm{T}} G\right)^{-1} G^{\mathrm{T}} x^{(1)}
$$

At last, second parameter fitting of GM $(1,1)$ model was got, combined Equation (7) with Equation (5).

\section{Markov Forecast Model}

Markov prediction is a kind of forecast method based on markov theory, suitable for stochastic volatility forecast problem. According to markov chain, the data sequence is divided into several states, $E_{1}, E_{2}, \cdots, E_{n}$ to represent. According to time sequence, we will transfer time off for $t_{1}, t_{2}, \cdots, t_{n}$, probability of sequence, which By $E_{i}$ after $k$ step into $E_{p}$ expressed by $p_{i j}^{(k)}$, that is

$$
p_{i j}^{(k)}=\frac{n_{i j}^{(k)}}{N_{i}}
$$

state $E_{i}$ after the number of $k$ step into $E_{p}$ while $N_{i}$ represent the total number of occurrence of state $E_{i}$ So, state transition probability matrix of $K$ steps is

$$
R^{(k)}=\left(\begin{array}{cccc}
p_{11}^{(k)} & p_{12}^{(k)} & \cdots & p_{1 j}^{(k)} \\
p_{21}^{(k)} & p_{22}^{(k)} & \cdots & p_{2 j}^{(k)} \\
\vdots & \vdots & \ddots & \vdots \\
p_{j 1}^{(k)} & p_{j 2}^{(k)} & \cdots & p_{j j}^{(k)}
\end{array}\right)
$$

By means of state transfer probability matrix, we can determine the sequence in the variable of the state and its maximum probability $\max \left[p_{i j}^{(k)}\right]$, and iden- 
tify the next step of the variable. Then, according to the turning probability, we modified the forecast value by means of markov [14]. If the maximum probability of the matrix $R^{(k)}$ in the first $k$ lines, have two or more than two same or similar, changes in relative gas emission are hard to be predicted by $R^{(1)}$, so it is need that we investigate in such matrix like $R^{(2)}, R^{(3)}$ and so on, until it can determine the future changes in relative gas emission.

Because of the markov state transfer probability matrix has the ability of tracking variables random fluctuations and ineffectiveness, we combine the State transfer probability matrix and the organic combination of GM $(1,1)$ model to realize the complementary advantages, thus the prediction precision of the model can be improved.

\section{Prediction Model in the Application of the Gas Emission Forecast Instance}

\subsection{The Establishment of the Model}

There is a mine gas outburst seriously in Huaibei mining group Co., LTD whose safety is very serious, so that we cannot ignore the prediction and prevention of the mine gas. The relative mine gas emission from 1996 to 2008 is shown in Table 1 . And we analyze the model on the basis of the table.

1) We establish GM $(1,1)$ model for the original sequence $x^{0}(t)(t=1,2, \cdots, n)$,

$$
\hat{x}^{(1)}(k+1)=324.44839 \mathrm{e}^{0.031747 k}-313.84839
$$

Let be

$$
\hat{x}^{(0)}(k+1)=\hat{x}^{(1)}(k+1)-\hat{x}^{(1)}(k)
$$

trend fitting values and residual can be calculated by Equation (9) and Equation (10), which is

$$
\Delta x(i)=\hat{x}^{(0)}(i)-x(i),
$$

and the relative error is $\Delta p(i)=\frac{\Delta x(i)}{x^{(0)}(i)}$.

The calculation results are shown in Table 2.

The second parameter fitting for grey GM $(1,1)$ model is

$$
\hat{x}^{(1)}(k+1)=325.97679 \mathrm{e}^{0.031747 k}-315.72746 .
$$

At the same way we can also figure out residual error and relative error, which can be seen in Table 2 .

2) The model needs to be test with posterior error and qualified one can be used in extreme value forecast. After Markov correction posterior ratio $c=0.022$ and $p=1$, which can be seen in Table 3. So, the model with high precision proved that it can be used in forecast. And the Grey Markov fitting curve of coal mine gas emission in 12 months has been shown in Figure 1.

3) We divide quadratic fitting error of $\operatorname{GM}(1,1)$ forecast model according to different state and establish markov model. Because of the uncertainty of the 
state boundaries, this example uses the optimal algorithms when solving the state transition probability matrix [15]: At first, taking a set of critical value sequence generation whose residual has already been known into the Equation (8). Then, transfer probability matrix can be obtained and tested by known data either. At last, the one who with high coincidence rate can be chosen as transition probability matrix. In this case, residual sequence can be divided into four intervals. And its probability has been shown in Table 3.

The state of the residual sequence is shown in Table 4 in this case.

4) After the trend values can be obtained with quadratic fitting of GM $(1,1)$ model, we do Markov correction for the residual and get second fitting values. Next, we can get the second residual error and relative error, as shown in Table 2.

\subsection{Markov Forecast Model of Relative Coal Mine Gas Emission}

Mine gas emission in 2017 could be forecast by using the grey Markov model. And according to Equations (9) (10) and Table 4, we can get one step transition probability matrix of relative coal mine gas emission:

$$
R^{(1)}=\left(\begin{array}{cccc}
0 & 0 & 0.5 & 0.5 \\
0 & 0 & 0.33 & 0.67 \\
0 & 1 & 0 & 0 \\
0.5 & 0.25 & 0 & 0.25
\end{array}\right)
$$

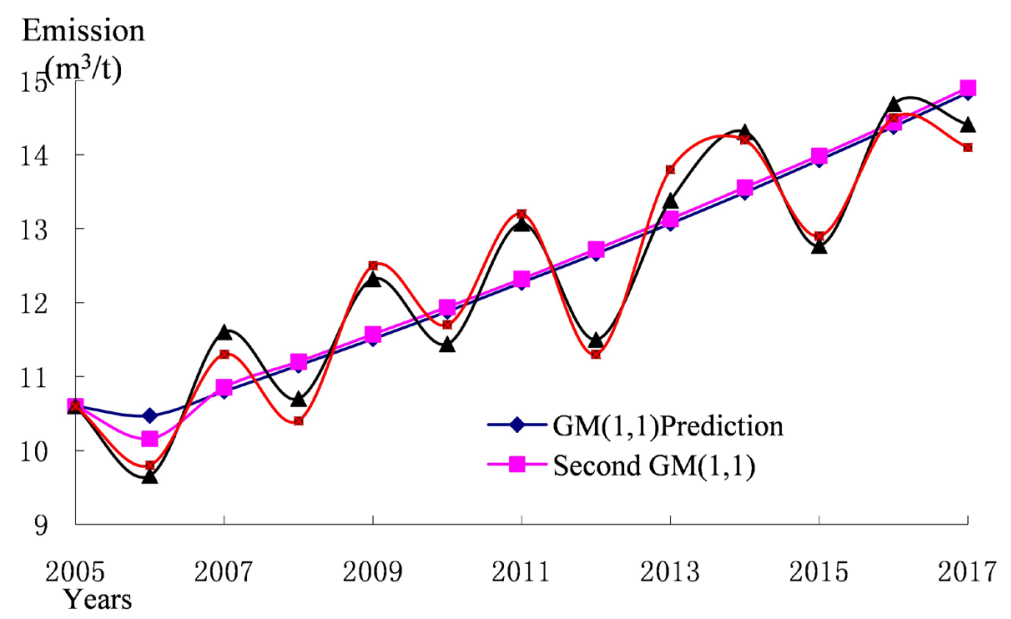

Figure 1. Mine years relative gas emission measurement and prediction chart.

Table 1. The mine gas emission inventory.

\begin{tabular}{cccccccc}
\hline years & 2005 & 2006 & 2007 & 2008 & 2009 & 2010 & 2011 \\
\hline $\begin{array}{c}\text { Gas emission } \\
\text { quantity }\left(\mathrm{m}^{3} / \mathrm{t}\right)\end{array}$ & 10.60 & 9.80 & 11.30 & 10.40 & 12.50 & 11.70 & 13.20 \\
\hline years & 2012 & 2013 & 2014 & 2015 & 2016 & 2017 & \\
\hline $\begin{array}{c}\text { Gas emission } \\
\text { quantity }\left(\mathrm{m}^{3} / \mathrm{t}\right)\end{array}$ & 11.30 & 13.80 & 14.20 & 12.90 & 14.50 & 14.90 & \\
\hline
\end{tabular}


Table 2. Prediction of grey markov model.

\begin{tabular}{|c|c|c|c|c|c|c|c|c|c|}
\hline \multirow{2}{*}{ Time } & \multirow{2}{*}{$\begin{array}{l}\text { Observed } \\
\text { values }\end{array}$} & \multicolumn{2}{|c|}{ GM $(1,1)$ model } & \multicolumn{3}{|c|}{$\begin{array}{l}\text { GM }(1,1) \text { model with the } \\
\text { second parameter fitting }\end{array}$} & \multicolumn{3}{|c|}{$\begin{array}{l}\text { GM }(1,1) \text { with second parameter } \\
\text { fitting-Markov model }\end{array}$} \\
\hline & & $\begin{array}{l}\text { Fitted values } \\
\qquad\left(\mathrm{m}^{3} / \mathrm{t}\right)\end{array}$ & $\begin{array}{l}\text { Relative } \\
\text { error \% }\end{array}$ & $\begin{array}{l}\text { Fitted value } \\
\qquad\left(\mathrm{m}^{3} / \mathrm{t}\right)\end{array}$ & $\begin{array}{l}\text { residual } \\
\left(\mathrm{m}^{3} / \mathrm{t}\right)\end{array}$ & $\begin{array}{l}\text { Relative } \\
\text { error \% }\end{array}$ & $\begin{array}{l}\text { Fitted value } \\
\qquad\left(\mathrm{m}^{3} / \mathrm{t}\right)\end{array}$ & $\begin{array}{l}\text { Residual } \\
\left(\mathrm{m}^{3} / \mathrm{t}\right)\end{array}$ & $\begin{array}{c}\text { Relative error } \\
\%\end{array}$ \\
\hline 2005 & 10.60 & 10.60 & 0.00 & 10.60 & 0.00 & 0.00 & 10.60 & 0 & 0.00 \\
\hline 2006 & 9.80 & 10.47 & -6.79 & 10.16 & -0.36 & -3.72 & 9.66 & 0.14 & 1.43 \\
\hline 2007 & 11.30 & 10.80 & 4.40 & 10.85 & 0.45 & 3.95 & 11.60 & -0.3 & -2.65 \\
\hline 2008 & 10.40 & 11.15 & -7.23 & 11.20 & -0.80 & -7.73 & 10.70 & -0.3 & -2.88 \\
\hline 2009 & 12.50 & 11.51 & 7.91 & 11.57 & 0.93 & 7.48 & 12.32 & 0.18 & 1.44 \\
\hline 2010 & 11.70 & 11.88 & -1.56 & 11.94 & -0.24 & -2.04 & 11.44 & 0.26 & 2.22 \\
\hline 2011 & 13.20 & 12.27 & 7.08 & 12.32 & 0.88 & 6.64 & 13.07 & 0.13 & 0.98 \\
\hline 2012 & 11.30 & 12.66 & -12.05 & 12.72 & -1.42 & -12.58 & 11.50 & -0.2 & -1.77 \\
\hline 2013 & 13.80 & 13.07 & 5.29 & 13.13 & 0.67 & 4.84 & 13.38 & 0.42 & 3.04 \\
\hline 2014 & 14.20 & 13.49 & 4.99 & 13.56 & 0.64 & 4.54 & 14.31 & -0.11 & -0.77 \\
\hline 2015 & 12.90 & 13.93 & -7.96 & 13.99 & -1.09 & -8.47 & 12.77 & 0.13 & 1.01 \\
\hline 2016 & 14.50 & 14.38 & 0.85 & 14.44 & 0.06 & 0.39 & 14.69 & -0.19 & -1.31 \\
\hline 2017 & 14.10 & 14.84 & -5.25 & 14.91 & -0.81 & -5.74 & 14.41 & -0.31 & -2.2 \\
\hline
\end{tabular}

Table 3. State probability partition.

\begin{tabular}{ccccc}
\hline State boundaries & {$[-1.45--1.00]$} & {$[-1.00-0.00]$} & {$[0.00-0.50]$} & {$[0.50-1.00]$} \\
\hline State & I & II & III & IV \\
\hline
\end{tabular}

Table 4. State of the residual sequence.

\begin{tabular}{ccccccccccccc}
\hline Years & 2005 & 2006 & 2007 & 2008 & 2009 & 2010 & 2011 & 2012 & 2013 & 2014 & 2015 & 2016 \\
\hline State & III & II & III & II & IV & II & IV & I & IV & IV & I & III \\
\hline
\end{tabular}

Because the relative mine gas emission in 2016 is $\mathrm{GM}(1,1)$ model prediction error III state, but $\max \left(p_{3 i}\right)=p_{32}$ so the relative gas emission error in this area in 2017 is most likely to turn to II state. According to Mark off revised forecast, the maximum possible value of the relative gas emission in this area in 2017 is $14.41 \mathrm{~m}^{3} / \mathrm{t}$ known when this area is known. The relative annual gas emission is $14.10 \mathrm{~m}^{3} / \mathrm{t}$, and the relative error is $-2.2 \%$. The prediction accuracy is fully consistent with the actual requirements.

\section{Conclusions}

In this paper, we established a grey Markov model to forecast the relative gas emission in Huaibei coal mine. And we got the conclusion as follows:

1) We combined grey forecast model with Markov model, and established grey Markov model to forecast mine gas emission. New model both has the advantages of grey model and Markov model. We can not only use less data estab- 
lishing model to forecast overall trend but also suitable for volatile random sequence forecast. The accuracy of new established model is significantly higher than the grey system GM $(1,1)$ model and the second parameter fitting of GM (1, 1) model.

2) We combined grey system GM $(1,1)$ forecast model and Markov chain to supply a new way to understand the characteristic of gas emission. Its advantage is the historical data can be fully used, and weakening many uncertain factors. So it can extend the application of grey system prediction and Markov chain prediction.

3) The proposed mine years relative gas emission prediction model also can be used to forecast different depth excavation working face gas emission and gas emission in the process of working face. And it has strong portability.

\section{Conflicts of Interest}

The authors declare no conflicts of interest regarding the publication of this paper.

\section{References}

[1] Zeng, Y. and Wu, C.F. (2004) Mine Gas Emission Prediction Fuzzy Points Realm and Research via the Network. Coal Science and Technology, 32, 62-65.

[2] Liu, C.R. and Ma, Y.D. (2005) Grey Prediction of Extending the Deep Gas Emission. The Mining Safety and Environmental Protection, 32, 1-4.

[3] Yu, Y. and Lu, J.G. (2006) The Gas Emission Forecast Based on the Grey Theory and Artificial Neural Network. Artificial Intelligence, 22, 269-272.

[4] Zhang, C.P. (2014) Application of Separate-Source Prediction Method on Gas Emission. Coal Technology, No. 10, 37-39.

[5] Si, R.J. (2014) Practice and Exploration of Gas Emission Rate Prediction in Zhaozhuang Mine. Shanxi Coal, No. 9, 23-25.

[6] Yuan, L. and Xue, S. (2014) Defining Outburst-Free Zones in Protective Mining with Seam Gas Content-Method and Application. Journal of China Coal Society, 39, 1786-1791.

[7] Liu, Y.W., Xue, W.T. and Li, Z.Q. (2015) Problems and Correction of Gas Emission Prediction Model in Mine Statistical Method. Safety in Coal Mines, 46, 176-178.

[8] Lv, G.C. and Ma, Y.D. (2004) Mine Gas Emission Prediction of Gray Modeling Method. Chinese Journal of Safety Science, 14, 22-25.

[9] Cao, A.H., Ding, Y.F., Jiang, S.G., Liu, T. and Zhang, P. (2012) Study on Multivariate Nonlinear Combination Prediction of Gas Emission. Safety in Coal Mines, 43, $1-4$.

[10] Zhao, X. and Song, G.J. (2012) Simulation Study on Nonlinear Forecasting Gas Emission Method. Computer Simulation, 29, 207-210.

[11] Wei, L., Fu, H. and Yin, Y.P. (2017) Nonlinear Dimension Reduction and Improved Elman Dynamic Prediction Model of Coal Mine Gas Emission. Journal of Liaoning Technical University (Natural Science), No. 4, 359-365.

[12] Deng, J.L. (1983) The Grey System (Social Economic). National Defence Industry Press, Beijing. 
[13] Ye, X.Y. and Yang, Y.J. (1994) Secondary Commercial Housing Price Forecast Model Parameter Fitting Grey Markov Chain. Science and Technology of Hydraulic and Electric Engineering, 21, 72-76.

[14] Yan, Y., Cheng, S.X. and Cheng, P. (1995) Operational Research Stochastic Model. China Republic University Public, Beijing.

[15] Knotters, M. and Van Walsum, P.E.V. (1997) Estimating Fluctuation Quantities from Time Series of Water-Table Depths Using Models with a Stochastic Component. Journal of Hydrology, 197, 25-46.

https://doi.org/10.1016/S0022-1694(96)03278-7 